\title{
Phosphorylation or mutation of the ERK2 activation loop alters oligonucleotide binding
}

\author{
Andrea C. McReynolds ${ }^{1, \ddagger}$, Aroon S. Karra ${ }^{1, \neq}$, Yan Li $^{1,5}$, Elias Daniel Lopez ${ }^{2}$, Adrian G. \\ Turjanski $^{2}$, Elhadji Dioum ${ }^{1,4}$, Kristina Lorenz $^{3}$, Elma Zaganjor ${ }^{1,6}$, Steve Stippec ${ }^{1}$, Kathleen $^{2}$ \\ McGlynn ${ }^{1}$, Svetlana Earnest ${ }^{1}$, and Melanie H. Cobb ${ }^{1,}{ }^{*}$ \\ ${ }^{1}$ Department of Pharmacology, UT Southwestern Medical Center, Dallas, TX \\ ${ }^{2}$ Laboratory of Structural Bioinformatics, Department of Chemical Biology, University of Buenos \\ Aires, Buenos Aires, Argentina \\ ${ }^{3}$ ISAS, Dortmund, Germany and ${ }^{5}$ NINDS Protein/Peptide Sequencing Facility, Bethesda, MD
}

\begin{abstract}
The mitogen-activated protein kinase ERK2 is able to elicit a wide range of context-specific responses to distinct stimuli, but the mechanisms underlying this versatility remain in question. Some cellular functions of ERK2 are mediated through regulation of gene expression. In addition to phosphorylating numerous transcriptional regulators, ERK2 is known to associate with chromatin and has been shown to bind oligonucleotides directly. ERK2 is activated by the upstream kinases MEK1/2, which phosphorylate both tyrosine 185 and threonine 183. ERK2 requires phosphorylation on both sites to be fully active. Some additional ERK2 phosphorylation sites have also been reported, including threonine 188. It has been suggested that this phosphoform has distinct properties. We detected some ERK2 phosphorylated on T188 in bacterial preparations of ERK2 by mass spectrometry and further demonstrate that phosphomimetic substitution of this ERK2 residue impairs its kinase activity toward well-defined substrates and also affects its DNA binding. We used electrophoretic mobility shift assays with oligonucleotides derived from the insulin gene promoter and other regions to examine effects of phosphorylation and mutations on ERK2 binding to DNA. We show that ERK2 can bind oligonucleotides directly. Phosphorylation and mutations alter DNA binding and support the idea that signaling functions may be influenced through an alternate phosphorylation site.
\end{abstract}

\section{Graphical Abstract}

\footnotetext{
${ }^{*}$ Corresponding Author: Melanie.Cobb@UTSouthwestern.edu; 214 645-6123.

${ }_{5}^{4}$ present address: Nestlé Institute, Lausanne, Switzerland

5 present address: NINDS

6 present address: Harvard Medical School

*A.C.M and A.S.K are co-first authors
}

Supporting Information Available: Figure 1 - Amino acid sequence alignment of kinase activation loops; Figure 2 - molecular dynamics simulation of T188 phosphorylation; Figure 3 - ERK2 oligonucleotide binding; Figure 4 - molecular model of ERK2 conformational changes from molecular dynamics; Figure 5- chromatin immunoprecipitation from H358 cells; Table 1 oligonucleotide sequences used in this study; Table 2 - gene regions containing oligonucleotides used in this study; supplementary detailed methods with references 


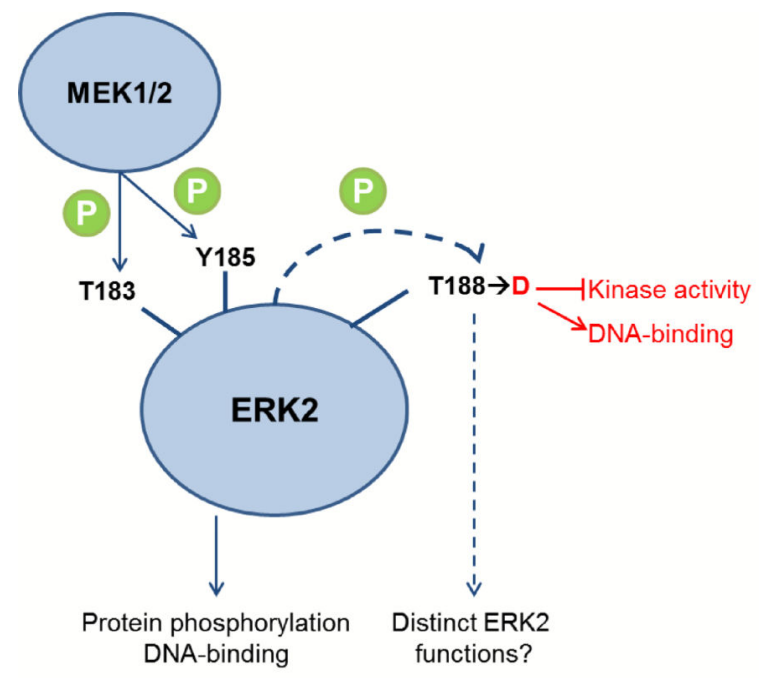

The mitogen-activated protein kinases (MAPKs) ERK1/2 mediate changes in cellular behavior by catalyzing the phosphorylation of hundreds of substrates throughout the cell in a context-specific manner ${ }^{1 ; 2}$. Substrates include plasma membrane receptors, ion transporters, cytoskeletal proteins, a variety of enzymes, and transcription factors ${ }^{3 ; 4}$. These kinases are activated by a broad range of cellular stimuli through a three-protein kinase cascade. Activation requires sequential phosphorylation on activation loop residues by the MAPK kinases (MAP2Ks) MEK1/2, first on Y185 (rat ERK2 numbering) and then on T183, to yield doubly phosphorylated ERK1/2 (ppERK2) with a $\mathrm{k}_{\text {cat }}$ increased as much as 50,000fold 5 .

Elevated circulating glucose activates ERK1/2 in pancreatic $\beta$ cells and increases association of glucose-regulated factors, including the kinases themselves, with the insulin gene promoter $^{6 ; 7}$. Chromatin immunoprecipitation (ChIP) experiments indicate that ERK1/2 activity is required to recruit and/or retain these factors on the insulin promoter in response to increased glucose. In these experiments we found that inhibition of ERK1/2 activation with MEK1/2 inhibitors decreased association of ERK1/2 with chromatin ${ }^{6}$. Several glucoseregulated transcription factors that induce insulin gene transcription are ERK1/2 substrates, including BETA2/NeuroD1, the basic helix-loop-helix protein partner, E47, and PDX-1 ${ }^{8}$. That all of these factors converge on a gene promoter suggests the activation-dependent phosphorylation of these transcription factors by active ERK1/2 on chromatin.

MAPKs can bind DNA directly in the absence of associated protein factors. In 1998, Bardwell, Thorner, and colleagues reported the direct binding of the yeast Kss1p MAPK to DNA $^{9}$. In $2009 \mathrm{Hu}$ et al reported the direct association of ERK2 with oligonucleotides and characterized a preferred binding site of $\mathrm{G} / \mathrm{GAAAG} / \mathrm{C}^{10}$, similar to the consensus identified for Kss1p. Authors of both of these studies concluded that MAPK binding resulted in transcriptional inhibition. Hu et al. provided evidence that unphosphorylated ERK2 inhibits gene transcription by preventing the interaction of C/EBP- $\beta$ with promoters of interferonregulated genes. Direct binding of ERK2 to DNA was also recently demonstrated using a similar oligonucleotide in a study of ERK1/2 on developmental promoters ${ }^{11}$. 
Several MAPK family members have also been studied in the context of cardiac hypertrophy ${ }^{12-15}$. Distinct functions of ERK2 in cardiac hypertrophy from those in normal cardiac function have recently been attributed to changes in kinase function resulting from its phosphorylation on a distinct site, T188, which lies near the well-described sites of activating phosphorylation, Y185 and T183 15 . Phosphorylation of T188 was found following stimulation of cells through a receptor tyrosine kinase and a $\mathrm{G}$ protein-coupled receptor. This combination of stimuli is thought to elicit a series of protein-protein interactions that promote autophosphorylation of activated ERK2 on Thr188 and activation of specific transcription factors in the nucleus.

To examine effects of phosphorylation on ERK2 binding to DNA, we tested oligonucleotide binding of recombinant ERK2 in vitro with and without phosphorylation on the activating sites. We also analyzed ERK2 expressed in bacteria by mass spectrometry to determine whether previously unrecognized modifications affect the oligonucleotide-binding capacity of ERK2. In the course of these studies, we identified fractions of ERK2 that were phosphorylated on T188. Based on a characterization of these ERK2 preparations and phosphomimetic mutants, we show that ERK2 activation enhances its ability to bind oligonucleotides and suggest that phosphorylation of T188, although inactivating, also enhances its interactions with oligonucleotides compared to the unphosphorylated state. These studies lend support to the idea that phosphorylation of ERK2, on the known regulatory sites and on T188, influences the output of the ERK1/2 MAPK pathway.

\section{Experimental Procedures}

\section{Materials}

The ERK2 antibody was as previously described ${ }^{16}$. The pT183/pY185 antibody (\#9106) was from Cell Signaling. The pT188 antibody was as described by Lorenz et al. ${ }^{15}$. The phosphatase MKP3 (SE-454) was purchased from Enzo Life Sciences. The phosphatases PP2A and PTP1 were purified as described ${ }^{17 ; 18}$. Myelin basic protein (MBP, M1891) was from Sigma.

\section{Rat ERK2 recombinant protein expression and purification}

Expression of recombinant ERK2 was generally as described previously ${ }^{19}$. For further details see Supplemental Methods.

\section{In vitro kinase assays}

ERK2 and mutants $(3 \mu \mathrm{M})$ were activated with $40-50 \mathrm{nM}$ constitutively active MEK1 (R4FMEK1) and $100 \mu \mathrm{M}$ ATP ([ $\left.\gamma^{32} \mathrm{P}-\mathrm{ATP}\right]$ 200-300 cpm/pmol) in $10 \mathrm{mM}$ Tris, $15 \mathrm{mM}$ $\mathrm{MgCl}_{2}, 1 \mathrm{mM}$ DTT, $1 \mathrm{mM}$ benzamidine for $30 \mathrm{~min}$ at $30^{\circ} \mathrm{C}$. ERK2 $(0.3 \mu \mathrm{M})$ activity was measured with $50 \mu \mathrm{g} / \mathrm{ml}$ myelin basic protein (MBP) and other substrates for 10 min under conditions above. Following gel electrophoresis and autoradiography, protein bands were excised and analyzed by scintillation counting. Reactions with peptide substrates were spotted onto $\mathrm{P} 81$ paper and washed $3 \times$ with $75 \mathrm{mM} \mathrm{H}_{3} \mathrm{PO}_{4}$ and then acetone, dried overnight, and counted as above. 


\section{Electrophoretic mobility shift assays (EMSAs)}

EMSAs were performed with both radioactively labeled oligonucleotides and a 5' labeled IRDye 700-tagged oligonucleotide (Integrated DNA Technologies) mapping to the rat insulin promoter. Briefly, oligonucleotides were incubated with purified ERK2 and mutants then resolved by non-denaturing polyacrylamide gel electrophoresis and imaged on a LICOR Odyssey system or by exposure to film. Initial DNA binding reactions were assembled by incubating ERK2 $(2.4 \mu \mathrm{M})$ in $10 \mathrm{mM}$ HEPES pH 7.4, $1 \mathrm{mM}$ dithiothreitol, $1 \mathrm{mM}$ benzamidine, $15 \mathrm{mM} \mathrm{MgCl} 2,0.5 \mu \mathrm{g} / \mathrm{ml}$ poly $\mathrm{dI}: \mathrm{dC}$ and fluorescently labeled oligonucleotide (Sigma) for $30 \mathrm{~min}$ at $30^{\circ} \mathrm{C}$ followed by an overnight incubation at $4{ }^{\circ} \mathrm{C}$. Reactions were separated on a $5 \%$ non-denaturing gel $(5 \%$ acrylamide, $50 \mathrm{mM}$ Tris $\mathrm{pH} 8.0,190 \mathrm{mM}$ glycine, $1.875 \mathrm{mM}$ EDTA, $15 \mathrm{mM} \mathrm{MgCl}_{2}$, and $5 \%$ glycerol) at $4{ }^{\circ} \mathrm{C}$ in $10 \mathrm{mM}$ Tris $\mathrm{pH} 8.0$, $400 \mathrm{mM}$ glycine and imaged on a LI-COR Odyssey imager. For titration experiments, reactions were for $30 \mathrm{~min}$ at room temperature then $16 \mathrm{~h}$ at $4^{\circ} \mathrm{C}$, but without benzamidine or poly dI:dC.

\section{Mass spectrometry}

Mass spectrometry was performed on ERK2 fractionated on MonoQ. Extracted peptides were analyzed by a nano-LC/MS/MS system with an Ultimate 3000 HPLC (ThermoDionex) connected to an Orbitrap Elite mass spectrometer (Thermo Scientific) via an EasySpray ion source (Thermo Scientific). Peptides were separated on ES800 Easy-Spray column ( $75 \mu \mathrm{m}$ inner diameter, $15 \mathrm{~cm}$ length, $3 \mu \mathrm{m} \mathrm{C18}$ beads; Thermo Scientific) at a flow rate of $300 \mathrm{nl} / \mathrm{min}$ with a $35 \mathrm{~min}$ linear gradient of $2-24 \%$ mobile phase B (mobile phase A: $2 \%$ acetonitrile, $0.1 \%$ formic acid; mobile phase B: $98 \%$ acetonitrile, $0.1 \%$ formic acid). Thermo Scientific Orbitrap Elite mass spectrometer was operated in positive nanoelectrospray mode. MS data were acquired in both profile and data dependent modes. The resolution of the survey scan was set at $60 \mathrm{k}$ at $\mathrm{m} / \mathrm{z} 400$ with a target value of 1'106 ions. The $\mathrm{m} / \mathrm{z}$ range for MS scans was 300-1600. The isolation window for MS/MS fragmentation was set to 1.9 and the top two most abundant ions were selected for product ion analysis. Ion trap enhanced scan rate was used for the MS/MS data acquisition with decision tree procedure activated. Dynamic exclusion was $12 \mathrm{~s}$ and early expiration was enabled. Xcalibur RAW files were converted to peak list files in mgf format using Mascot Distiller (version 2.4.3.3). Database search was performed using Mascot Daemon (2.4.0) against a house-built database containing NCBI human sequences and Tag+Erk2 sequence.

\section{Computational methods}

See Supplemental Methods

\section{Results}

\section{Purification of recombinant ERK2 and analysis by mass spectrometry}

A ribbon diagram of rat ERK2 is shown in Fig. 1A. Recombinant His $_{6}$-ERK2 was expressed and purified on nickel resin with little modification to previous protocols, yielding ERK2 with an estimated purity of $65 \%$. Protein was further purified on MonoQ. Equal amounts of protein from each fraction were assayed for kinase activity (Fig. 1B). Activity was detected 
near the tail of the elution peaks (not shown) with more total activity at the end of the first peak than the second, similar to what was described previously by Prowse and Lew ${ }^{20}$.

Pooled fractions were analyzed by mass spectrometry. Multiple phosphorylated species were detected, including phosphorylated Y185 (pY185), phosphorylated T183 (pT183), and phosphorylated T188 (pT188) (Fig. 1A), as shown by the spectra (Fig. 1C-E).

To determine if phosphorylation of T188 was influencing the ion-exchange elution profile, mass spectrometry was performed on the fractions from another MonoQ separation (Fig.

2A). Many fractions contained pY185 and pT183, but the low yield of pT188 indicates that only some of the ERK2 in each fraction was phosphorylated on this site.

Conserved residues in the ERK2 catalytic and activation loops including T188 create the P +1 pocket, a binding site for the residue of protein substrates that is on the C-terminal side of the phosphorylation site $(\mathrm{P}+1$ residue) (Fig. 1A). Alignments of protein kinase sequences indicate that threonine is highly conserved in this position (Supplementary Fig. 1). In 2009 phosphorylation of ERK2 on T188 was identified from phosphopeptide maps that revealed a unique peptide from ERK2 incubated with heterotrimeric G protein $\beta \gamma$ subunits compared to ERK2 incubated only with upstream kinase cascade components ${ }^{15}$. The novel site in the phosphopeptide was identified by mutagenesis as pT188 and was suggested to arise by autophosphorylation. Using a pT188-specific antibody ${ }^{15}$, we detected this phospho-form in multiple MonoQ fractions and in a pooled preparation of wild type protein but not in fractions of the inactive ERK2 mutant (K52R) (Fig. 2B and 2C). Notably, treatment with the MAPK phosphatase MKP3 did not dephosphorylate pT188 despite removing phosphate from the activating sites, as determined by immunoblotting with an antibody specific for ERK2 phosphorylated on T183 and Y185 (active or ppERK2) and an antibody which recognizes total ERK2 (independent of its phosphorylation state) (Fig. 2B). Mutation of T188 to alanine inactivates ERK2 ${ }^{21}$, and mutation of the comparable residue in some other protein kinases is inactivating as well ${ }^{22 ; 23}$, leading us to hypothesize that ERK2 phosphorylated on T188 is inactive. This ERK2 phospho-form was probably not detected earlier because it is a minor component in bacterial preparations, consistent with our finding that pT188-containing fractions still harbor kinase activity towards MBP upon activation by constitutively active MEK1 (MEK1-R4F) (Fig. 2D).

To model how autophosphorylation of T188 might occur, molecular dynamics was performed with the expectation that the target residues must come within $4 \AA$ of the gamma phosphate of bound ATP (Fig. 3E and Supplementary Fig. 4). Structural studies on ERK2 indicate that the T188 hydroxyl group is within $6 \AA$ of the gamma phosphate group, and umbrella sampling reveals ERK2 phosphorylated on Y185 is energetically stable with the distance reduced to $4 \AA$. Molecular modeling suggests that the ERK2 activation loop can sustain triple phosphorylation events and also that autophosphorylation of ERK2 on T188 is most likely to occur after phosphorylation of ERK2 on Y185 but prior to its phosphorylation on T183 (Fig. 2E, Fig. 3E, Supplementary Fig. 2).

\section{Activities of ERK2 and mutants}

ERK2 fractions containing pT188 can be phosphorylated and activated by constitutively active MEK1 (MEK1R4F) similarly to unphosphorylated protein. Because ERK2 pT188 
was a minor component of each fraction, we could not determine unequivocally whether or not ERK2 pT188 was active. This form proved difficult to purify due to the heterogeneous mixture of trace phosphorylations. Instead, we expressed the ERK2 mutants, T188D and T188E, as phosphomimetic substitutions. Expression and solubility of ERK2 T188D and ERK2 T188E were both similar to wild type ERK2. We compared the protein kinase activities of ERK2 wild type, T188D, and T188E toward the model substrate MBP and found that wild type ERK2 had readily detectable activity, but ERK2 T188D and T188E did not (Fig. 3A).

To test whether this was a consequence of mis-folding, we phosphorylated these proteins with MEK1R4F in vitro. MEK1 binds at least two regions on ERK2: the common docking (CD) loop and the MAPK insert ${ }^{24-26}$, the latter of which is required for ERK activation by MEK. The T188 mutants were both phosphorylated roughly as well as wild type ERK2, while the inactive ERK2 mutant Y185F (inactive because it lacks the initial MEK phosphorylation $\operatorname{sit}^{27}$ ) was not (Fig. 3B). Immunoblotting with the active ERK2 antibody shows that phosphorylation of ERK2 and T188 mutants was comparable, indicating that the T188 mutants were well-folded. Although the T188 mutants did not harbor activity towards the standard model substrate MBP, we considered the idea that changes in the orientation of the $\mathrm{P}+1$ pocket might alter its substrate specificity. We tested several additional substrates including kinase-dead MEK1 (MEK1KM), nucleoporin 153 (Nup153), the insulin gene transcription factor PDX-1, and the peptide ERKtide, taken from the sequence of tyrosine hydroxylase ${ }^{8 ; 28-30}$ (Fig. 3C and 3D). Activity towards the protein substrates was not detected, but a small amount of ERK2T188E activity was detected with ERKtide, perhaps due to greater flexibility of a small peptide compared to full-length protein substrates (Fig. $3 \mathrm{D})$,

\section{ERK2 binds oligonucleotides derived from several gene promoters}

A range of experiments has identified multiple genes with which ERK1/2 may associate $6 ; 10 ; 11 ; 31 ; 32$. A subset of genes that ERK2 binds (Supplementary Fig. 5), as confirmed by chromatin immunoprecipitation (ChIP), are listed in Supplementary Table 2. These include growth hormone (GH), unc-like kinase 1 (Ulk1), insulin-like growth factor binding protein 2 (IGFBP2), chromatin regulating lysine demethylase 2a (KDM2a-2), and the phosphatase eyes absent 1 (EYA-1). Inhibition of pathway activation by the MEK inhibitor PD0325901 decreased association of ERK2 with each of these genes in the ChIP assays. Oligonucleotides were synthesized derived from the ERK2-binding peak of these genes and used for electrophoretic mobility shift assays (EMSAs) (Supplementary Fig. 3A and Fig. 4). The oligonucleotides each contained the ERK2 binding consensus G/ CAAAG/C, except for that from KDM2a. Oligonucleotides derived from ULK1 and EYA1 gene promoters each bound active ERK2. Less binding was observed to an equivalent amount of ERK2 T183E or Y185F. The EYA-1 oligonucleotide bound almost equally well to Y185F ERK2. In contrast, the oligonucleotide from KDM2a, which lacks the G/ CAAAG/C consensus, bound less ERK2 than other oligonucleotides tested (Supplementary Table 1). Importantly, phosphorylated ERK2 (ppERK2) bound DNA more robustly than ERK2 that has not been activated with MEK1R4F (Fig. 4). 


\section{ERK2 and activation loop mutants bind directly to an insulin gene promoter sequence}

We showed previously that ERK1/2 bind to the insulin gene promoter in pancreatic beta cells and that the interaction is strongly enhanced if the kinases are active ${ }^{6 ; 7}$. His $_{6}$-ERK2 was co-expressed with MEK1R4F to generate quantities of active ERK2 phosphorylated on Y185 and T183 to compare to ERK2 and mutants. Synthetic oligonucleotides representing $47 \mathrm{bp}$ and $19 \mathrm{bp}$ sequences from the mouse insulin gene promoter were used that contain the sequence G/GAAAG/C, previously identified as a consensus recognized by ERK2 and the yeast MAPK Kss1p ${ }^{9 ; 10}$ (Fig. 4 and Supplementary Fig. 3B). To assess the relative DNAbinding ability of ERK2 upon perturbation of its activation loop better, we employed a fluorescently labeled version of the $19 \mathrm{bp}$ insulin promoter sequence in gel shift assays over a range of protein concentrations (Fig. 4B and D). Wild type ERK2 binds the probe at the highest concentrations tested. Activated ERK2 and activation loop mutants, e.g., T188D, shift the probe at lower concentrations (Fig. 4D). This suggests that activation of ERK2 increases its ability to bind DNA. Interestingly, eliminating a potential hydrogen bond by mutating T188 to the nearly isosteric amino acid valine also alters the DNA binding activity of ERK2 relative to wild type protein, highlighting the sensitivity of this residue in ERK2DNA interactions (Fig. 4D).

\section{Discussion}

The activation loop of ERK2 makes critical contacts with the MAPK insert that are destabilized upon activating phosphorylation, thereby altering accessibility of the insert. The greater accessibility of the MAPK insert accounts for increased binding of active ERK2 to FXF-containing docking motifs present on many of its substrates ${ }^{33}$. The MAPK insert region of ERK2 has also been implicated by mutagenesis as the portion of ERK2 that contacts the DNA consensus sequence ${ }^{10}$. Hu et al. suggested that unphosphorylated ERK2 binds the G/CAAAG/C consensus. We also find that ERK2 binds this consensus and that phosphorylated ERK2 binds more strongly. Intriguingly, direct interactions of DNA with prokaryotic kinases have also been described, such as the bacterial persistence factor HipA, which possesses a eukaryotic kinase fold ${ }^{34}$. In complex with the DNA-binding neutralizing protein HipB, HipA contacts DNA through a region of the molecule similar to the MAPK insert in ERK2. The MAPK insert is present in members of the CGMC branch of the kinome, including GSK3, cyclin-dependent kinases, and yeast Kss1p, all of which are known to associate with chromatin and regulate gene expression 9;35;36. Moreover, it is conceivable that multiple members of this kinase cohort with positively charged insert regions can associate with DNA through this structural element.

Alignments of eukaryotic protein kinases reveal a highly conserved threonine at the position comparable to T188 in ERK2, which forms part of the $\mathrm{P}+1$ pocket $^{37}$. The flexibility of the $\mathrm{P}$ +1 pocket contributes to substrate binding, positioning, and release. T188 is part of a hydrogen-bonding network within the ERK2 core, as elaborated upon by Emrick et al. ${ }^{21}$. Mutation of this threonine in ERK2 and the comparable residue in other protein kinases has been previously shown to yield inactive enzymes ${ }^{21 ; 22}$, consistent with our findings here. A logical assumption is that this residue is not regulated by phosphorylation. Nevertheless, in the kinase MARK1, phosphorylation of the comparable residue (S212) was detected in brain 
lysates using a phospho-specific antibody, similar to the observation of ERK2 pT188 in a cardiac hypertrophy model ${ }^{15 ; 22}$.

As isolated from bacteria, ERK2 is largely unphosphorylated but contains a heterogeneous background of phosphorylation states, including detectable phosphorylation on the well known activating sites, Y185 and T183. Surprisingly, we also found ERK2 phosphorylated on T188 in this heterogeneous array. ERK2 is capable of autophosphorylation not only on $\mathrm{Y} 185^{38}$, but, at least under certain circumstances, also on $\mathrm{T} 183$ and $\mathrm{T} 188$. The prediction from molecular dynamics is that the ERK2 activation loop can support all three phosphorylations with ERK2 T188 autophosphorylation following that on Y185, paralleling the experimentally derived sequence of events proposed by Lorenz et al. ${ }^{15}$. If the MAPK insert is indeed the site of DNA binding, modification of T188 may also facilitate the DNA accessibility of ERK2 on this region.

The physiological relevance of phosphorylation of T188 is not known apart from its proposed role in cardiac function, but further work has suggested that preventing phosphorylation of ERK2 on T188 by mutagenesis reduces pathological cardiac hypertrophy ${ }^{39}$. The inability of ERK2 T188 phosphomimetic mutants to phosphorylate the various substrates that we tested suggests that the substrate recognition groove, as well as critical contacts in the active site, are altered, rendering the enzyme inactive. However, although this work suggests that perturbation of T188 results in inactivity toward known substrates, it remains possible that phosphorylation of T188 confers on ERK2 the ability to phosphorylate distinct substrates. A precedent for this within the MAPK family comes from the work of Ashwell and colleagues who have demonstrated that an alternative form of activated $\mathrm{p} 38$ has distinct substrate specificity in T cells ${ }^{40}$. This alternative state is induced by a newly identified C-terminal phosphorylation event that causes refolding of the protein substrate binding region.

ERK2 has a variety of functions in regulating transcription, many of which may be aided by the direct association of ERK2 with genes. ERK2 is in excess of most transcriptional regulators at a cellular concentration estimated to be greater than $100 \mathrm{nM}$, and perhaps much higher based on recently reported concentrations of C-Raf and MEK1 in the micromolar range ${ }^{41}$. Given the polyelectrolytic nature of DNA, low affinity interactions between an exposed duplex and proteins are primarily driven by electrostatics. Nevertheless, beyond the generalized affinity of a nuclear regulator such as ERK2 for chromatin, phosphorylation events can drive conformations toward more specific interactions, similar to what has been observed in prokaryotic systems, as discussed by von Hippel ${ }^{42}$. The consensus binding site may offer a relatively low affinity depot of ERK2 to regulate nearby chromatin bound factors, many of which are capable of generating higher affinity sites on chromatin through scaffolds and substrate proteins ${ }^{43}$.

ERK1/2 promoter residency is not limited to the insulin gene promoter ${ }^{6}$. ERK1/2 and multiple upstream kinases have been studied on the Egr1 promoter ${ }^{32}$, and widespread MAPK promoter binding has been suggested through several studies in yeast and mammalian cells ${ }^{44}$. Extensive interactions of Hog1p, a yeast p38 MAPK, with chromatin have been documented ${ }^{45 ; 6}$, and more recently, ERK1/2 were shown to be recruited to 
estrogen response elements along with estrogen receptor $A^{47}$. ERK1/2 have also been found to occupy promoters of numerous genes in stem cells ${ }^{48}$. Given the prominence of phosphorylation in controlling gene expression ${ }^{49}$, the malleable DNA-binding ability of ERK2 may be critical for modulating the signaling output of this pathway. ERK2 phosphorylation states with different effects on DNA binding indicate a necessary focus of future work in this area, especially as it pertains to the promotion of disease. The possibility of widespread ERK1/2-DNA binding and potential regulation of gene expression or alternative signaling has implications for both kinase biology and chromatin regulation.

\section{Supplementary Material}

Refer to Web version on PubMed Central for supplementary material.

\section{Acknowledgments}

These studies were supported by National Institutes of Health grant R37 DK34128 and Welch Foundation grant I1243 to MHC and Deutsche Forschungsgemeinschaft (DFG; Sonderforschungsbereich SFB688) to KL.

The authors thank Michael Kalwat, Aileen Klein, Hashem Dbouk, Clinton Taylor and other current and former members of the Cobb laboratory for valuable discussions, Nida Iqbal for help with figures, and Dionne Ware for administrative assistance.

\section{Abbreviations used in text}

BETA2/NeuroD1

CD

ChIP

DTT

EDTA

EGTA

ERK

EYA-1

GH1

HipA

HipB

Hog1p

HPLC

IGFBP2

IPTG $\beta 2$-adrenergic agonist $2 /$ Neurogenic differentiation 1

common docking

chromatin immunoprecipitation

dithiothreitol

ethylenediaminetetraacetic acid

ethylene glycol tetraacetic acid

extracellular signal-regulated kinase

eyes absent homolog 1

growth hormone 1

high-persistance A

high-persistance B

high osmolarity glycerol-response

high performance liquid chromatography

insulin-like growth factor binding protein 2

isopropyl $\beta$-D-1-thiogalactopyranoside 


$\begin{array}{ll}\text { IRDye } & \text { infrared dye } \\ \text { KDM2a } & \text { lysine demethylase 2a } \\ \text { Kss1p } & \text { kinase suppressor of Sst2 mutations } \\ \text { LC/MS/MS } & \begin{array}{l}\text { liquid chromatography/mass spectrometry/mass } \\ \text { spectrometry }\end{array} \\ \text { MAP2K } & \text { mitogen-activated protein kinase kinase } \\ \text { MAPK } & \text { mitogen-activated protein kinase } \\ \text { MBP } & \text { myelin basic protein } \\ \text { MD } & \text { molecular dynamics } \\ \text { MEK } & \text { mitogen/extracellular signal-regulated kinase } \\ \text { mIns } & \text { mouse insulin } \\ \text { MKP3 } & \text { MAP kinase phosphatase 3 } \\ \text { Nup153 } & \text { nucleoporin 153 } \\ \text { PDX-1 } & \text { pancreatic and duodenal homeobox 1 } \\ \text { PP2A } & \text { protein phosphatase 2 } \\ \text { PTP1 } & \text { protein tyrosine phosphatase } \\ \text { Ulk1 } & \text { unc-51 like autophagy activating kinase 1 }\end{array}$

\section{References}

1. Cargnello M, Roux PP. Activation and function of the MAPKs and their substrates, the MAPKactivated protein kinases. Microbiol. Mol. Biol. Rev. 2011; 75(1):50-83. [PubMed: 21372320]

2. Raman M, Chen W, Cobb MH. Differential regulation and properties of MAPKs. Oncogene. 2007; 26(22):3100-3112. [PubMed: 17496909]

3. Carlson SM, Chouinard CR, Labadorf A, Lam CJ, Schmelzle K, Fraenkel E, White FM. Large-scale discovery of ERK2 substrates identifies ERK-mediated transcriptional regulation by ETV3. Sci. Signal. 2011; 4(196):rs11. [PubMed: 22028470]

4. Courcelles M, Fremin C, Voisin L, Lemieux S, Meloche S, Thibault P. Phosphoproteome dynamics reveal novel ERK1/2 MAP kinase substrates with broad spectrum of functions. Mol. Syst. Biol. 2013; 9:669. [PubMed: 23712012]

5. Prowse CN, Deal MS, Lew J. The complete pathway for catalytic activation of the mitogen-activated protein kinase, ERK2. J. Biol. Chem. 2001; 276:40817-40823. [PubMed: 11524422]

6. Lawrence MC, McGlynn K, Shao C, Duan L, Naziruddin B, Levy MF, Cobb MH. Chromatin-bound mitogen-activated protein kinases transmit dynamic signals in transcription complexes in $\{$ beta $\}$ cells. Proc. Natl. Acad. Sci. U. S. A. 2008; 105:13315-13320. [PubMed: 18755896]

7. Lawrence MC, Shao C, McGlynn K, Naziruddin B, Levy MF, Cobb MH. Multiple chromatin-bound protein kinases assemble factors that regulate insulin gene transcription. Proc. Natl. Acad. Sci. USA. 2009; 106(52):22181-22186. [PubMed: 20018749] 
8. Khoo S, Griffen SC, Xia Y, Baer R, German MS, Cobb MH. Regulation of insulin gene transcription by extracellular-signal regulated protein kinases (ERK) 1 and 2 in pancreatic beta cells. J. Biol. Chem. 2003; 278:32969-32977. [PubMed: 12810726]

9. Bardwell L, Cook JG, Voora D, Baggott DM, Martinez AR, Thorner J. Repression of yeast Ste12 transcription factor by direct binding of unphosphorylated Kss1 MAPK and its regulation by the Ste7 MEK. Genes Dev. 1998; 12(18):2887-2898. [PubMed: 9744865]

10. Hu S, Xie Z, Onishi A, Yu X, Jiang L, Lin J, Rho HS, Woodard C, Wang H, Jeong JS, Long S, He X, Wade H, Blackshaw S, Qian J, Zhu H. Profiling the human protein-DNA interactome reveals ERK2 as a transcriptional repressor of interferon signaling. Cell. 2009; 139(3):610-622. [PubMed: 19879846]

11. Tee WW, Shen SS, Oksuz O, Narendra V, Reinberg D. Erk1/2 activity promotes chromatin features and RNAPII phosphorylation at developmental promoters in mouse ESCs. Cell. 2014; 156(4):678690. [PubMed: 24529373]

12. Montessuit $\mathrm{C}$, Thorburn A. Transcriptional activation of the glucose transporter GLUT1 in ventricular cardiac myocytes by hypertrophic agonists. J Biol Chem. 1999; 274(13):9006-9012. [PubMed: 10085148]

13. Aoki H, Richmond M, Izumo S, Sadoshima J. Specific role of the extracellular signal-regulated kinase pathway in angiotensin II-induced cardiac hypertrophy in vitro. Biochem. J. 2000; 347(Pt 1):275-284. [PubMed: 10727428]

14. Nicol RL, Frey N, Pearson G, Cobb M, Richardson J, Olson EN. Activated MEK5 induces serial assembly of sarcomeres and eccentric cardiac hypertrophy. EMBO J. 2001; 20(11):2757-2767. [PubMed: 11387209]

15. Lorenz K, Schmitt JP, Schmitteckert EM, Lohse MJ. A new type of ERK1/2 autophosphorylation causes cardiac hypertrophy. Nat. Med. 2009; 15(1):75-83. [PubMed: 19060905]

16. Boulton TG, Cobb MH. Identification of multiple extracellular signal-regulated kinases (ERKs) with antipeptide antibodies. Cell Regulation. 1991; 2:357-371. [PubMed: 1654126]

17. Mumby MC, Green DD, Russell KL. Structural characterization of cardiac protein phosphatase with a monoclonal antibody. Evidence that the $\mathrm{M}_{\mathrm{r}}=38,000$ phosphatase is the catalytic subunit of the native enzyme(s). J. Biol. Chem. 1985; 260:13763-13770. [PubMed: 2997181]

18. Guan K, Dixon JE. Evidence for protein-tyrosine-phosphatase catalysis proceeding via a cysteinephosphate intermediate. J. Biol. Chem. 1991; 266(26):17026-17030. [PubMed: 1654322]

19. Wilsbacher JL, Goldsmith EJ, Cobb MH. Phosphorylation of MAP kinases by MAP/ERK kinases involves multiple regions of the MAP kinases. J. Biol. Chem. 1999 Jun 11.274:16988-16994. [PubMed: 10358048]

20. Prowse CN, Lew J. Mechanism of activation of ERK2 by dual phosphorylation. J. Biol. Chem. 2001; 276(1):99-103. [PubMed: 11016942]

21. Emrick MA, Lee T, Starkey PJ, Mumby MC, Resing KA, Ahn NG. The gatekeeper residue controls autoactivation of ERK2 via a pathway of intramolecular connectivity. Proc. Natl. Acad. Sci. U. S. A. 2006; 103(48):18101-18106. [PubMed: 17114285]

22. Timm T, Li XY, Biernat J, Jiao J, Mandelkow E, Vandekerckhove J, Mandelkow EM. MARKK, a Ste20-like kinase, activates the polarity-inducing kinase MARK/PAR-1. EMBO J. 2003; 22(19): 5090-5101. [PubMed: 14517247]

23. Pike AC, Rellos P, Niesen FH, Turnbull A, Oliver AW, Parker SA, Turk BE, Pearl LH, Knapp S. Activation segment dimerization: a mechanism for kinase autophosphorylation of non-consensus sites. EMBO J. 2008; 27(4):704-714. [PubMed: 18239682]

24. Tanoue T, Adachi M, Moriguchi T, Nishida E. A conserved docking motif in MAP kinases common to substrates, activators and regulators. Nat. Cell Biol. 2000; 2(2):110-116. [PubMed: $10655591]$

25. Xu B, Wilsbacher JL, Collisson T, Cobb MH. The N-terminal ERK binding site of MEK1 is required for efficient feedback phosphorylation by ERK2 in vitro and ERK activation in vivo. J. Biol. Chem. 1999; 274(48):34029-34035. [PubMed: 10567369]

26. Robinson FL, Whitehurst AW, Raman M, Cobb MH. Identification of novel point mutations in ERK2 that selectively disrupt binding to MEK1. J Biol. Chem. 2002; 277(17):14844-14852. [PubMed: 11823456] 
27. Robbins DJ, Zhen E, Owaki H, Vanderbilt C, Ebert D, Geppert TD, Cobb MH. Regulation and properties of extracellular signal-regulated protein kinases 1 and 2 in vitro. J. Biol. Chem. 1993; 268:5097-5106. [PubMed: 8444886]

28. Whitehurst AW, Wilsbacher JL, You Y, Luby-Phelps K, Moore MS, Cobb MH. ERK2 enters the nucleus by a carrier-independent mechanism. Proc. Natl. Acad. Sci. U. S. A. 2002; 99(11):74967501. [PubMed: 12032311]

29. Kosako H, Yamaguchi N, Aranami C, Ushiyama M, Kose S, Imamoto N, Taniguchi H, Nishida E, Hattori S. Phosphoproteomics reveals new ERK MAP kinase targets and links ERK to nucleoporin-mediated nuclear transport. Nat. Struct. Mol. Biol. 2009; 16(10):1026-1035. [PubMed: 19767751]

30. Haycock JW, Ahn NG, Cobb MH, Krebs EG. ERK1 and ERK2, Two Microtubule-associated Protein 2 Kinases, Mediate the Phosphorylation of Tyrosine Hydroxylase at Serine-31 in situ. Proc. Natl. Acad. Sci. USA. 1992; 89:2365-2369. [PubMed: 1347949]

31. Nelson JD, LeBoeuf RC, Bomsztyk K. Direct recruitment of insulin receptor and ERK signaling cascade to insulin-inducible gene loci. Diabetes. 2011; 60(1):127-137. [PubMed: 20929976]

32. Mikula M, Bomsztyk K. Direct recruitment of ERK cascade components to inducible genes is regulated by heterogeneous nuclear ribonucleoprotein (hnRNP) K. J. Biol. Chem. 2011; 286(11): 9763-9775. [PubMed: 21233203]

33. Lee T, Hoofnagle AN, Kabuyama Y, Stroud J, Min X, Goldsmith EJ, Chen L, Resing KA, Ahn NG. Docking motif interactions in MAP kinases revealed by hydrogen exchange mass spectrometry. Mol. Cell. 2004; 14(1):43-55. [PubMed: 15068802]

34. Schumacher MA, Piro KM, Xu W, Hansen S, Lewis K, Brennan RG. Molecular mechanisms of HipA-mediated multidrug tolerance and its neutralization by HipB. Science. 2009; 323(5912): 396-401. [PubMed: 19150849]

35. Vincent T, Kukalev A, Andang M, Pettersson R, Percipalle P. The glycogen synthase kinase (GSK) 3beta represses RNA polymerase I transcription. Oncogene. 2008; 27(39):5254-5259. [PubMed: 18490923]

36. Narayanan R, Adigun AA, Edwards DP, Weigel NL. Cyclin-dependent kinase activity is required for progesterone receptor function: novel role for cyclin $\mathrm{A} / \mathrm{Cdk} 2$ as a progesterone receptor coactivator. Mol. Cell Biol. 2005; 25(1):264-277. [PubMed: 15601848]

37. Goldsmith EJ, Akella R, Min X, Zhou T, Humphreys JM. Substrate and docking interactions in serine/threonine protein kinases. Chem Rev. 2007; 107(11):5065-5081. [PubMed: 17949044]

38. Haystead TAJ, Dent P, Wu J, Haystead CMM, Sturgill TW. Ordered phosphorylation of p42 mapk by MAP kinase kinase. FEBS Lett. 1992; 306:17-22. [PubMed: 1628739]

39. Ruppert C, Deiss K, Herrmann S, Vidal M, Oezkur M, Gorski A, Weidemann F, Lohse MJ, Lorenz K. Interference with ERK(Thr188) phosphorylation impairs pathological but not physiological cardiac hypertrophy. Proc. Natl. Acad. Sci. U. S. A. 2013; 110(18):7440-7445. [PubMed: 23589880]

40. Salvador JM, Mittelstadt PR, Guszczynski T, Copeland TD, Yamaguchi H, Appella E, Fornace AJ $\mathrm{Jr}$, Ashwell JD. Alternative $\mathrm{p} 38$ activation pathway mediated by $\mathrm{T}$ cell receptor-proximal tyrosine kinases. Nat. Immunol. 2005; 6(4):390-395. [PubMed: 15735648]

41. Romano D, Nguyen LK, Matallanas D, Halasz M, Doherty C, Kholodenko BN, Kolch W. Protein interaction switches coordinate Raf-1 and MST2/Hippo signalling. Nat. Cell Biol. 2014; 16(7): 673-684. [PubMed: 24929361]

42. von Hippel PH. From "simple" DNA-protein interactions to the macromolecular machines of gene expression. Annu. Rev. Biophys. Biomol. Struct. 2007; 36:79-105. [PubMed: 17477836]

43. Ruthenburg AJ, Li H, Milne TA, Dewell S, McGinty RK, Yuen M, Ueberheide B, Dou Y, Muir TW, Patel DJ, Allis CD. Recognition of a mononucleosomal histone modification pattern by BPTF via multivalent interactions. Cell. 2011; 145(5):692-706. [PubMed: 21596426]

44. Edmunds JW, Mahadevan LC. MAP kinases as structural adaptors and enzymatic activators in transcription complexes. J. Cell Sci. 2004; 117(Pt 17):3715-3723. [PubMed: 15286173]

45. Pokholok DK, Zeitlinger J, Hannett NM, Reynolds DB, Young RA. Activated signal transduction kinases frequently occupy target genes. Science. 2006; 313(5786):533-536. [PubMed: 16873666] 
46. Proft M, Mas G, De Nadal E, Vendrell A, Noriega N, Struhl K, Posas F. The stress-activated Hog1 kinase is a selective transcriptional elongation factor for genes responding to osmotic stress. Mol. Cell. 2006; 23(2):241-250. [PubMed: 16857590]

47. Madak-Erdogan Z, Lupien M, Stossi F, Brown M, Katzenellenbogen BS. Genomic collaboration of estrogen receptor alpha and extracellular signal-regulated kinase 2 in regulating gene and proliferation programs. Mol. Cell Biol. 2011; 31(1):226-236. [PubMed: 20956553]

48. Goke J, Chan YS, Yan J, Vingron M, Ng HH. Genome-wide kinase-chromatin interactions reveal the regulatory network of ERK signaling in human embryonic stem cells. Mol. Cell. 2013; 50(6): 844-855. [PubMed: 23727019]

49. Govind CK, Qiu H, Ginsburg DS, Ruan C, Hofmeyer K, Hu C, Swaminathan V, Workman JL, Li B, Hinnebusch AG. Phosphorylated Pol II CTD recruits multiple HDACs, including Rpd3C(S), for methylation-dependent deacetylation of ORF nucleosomes. Mol. Cell. 2010; 39(2):234-246.

[PubMed: 20670892] 


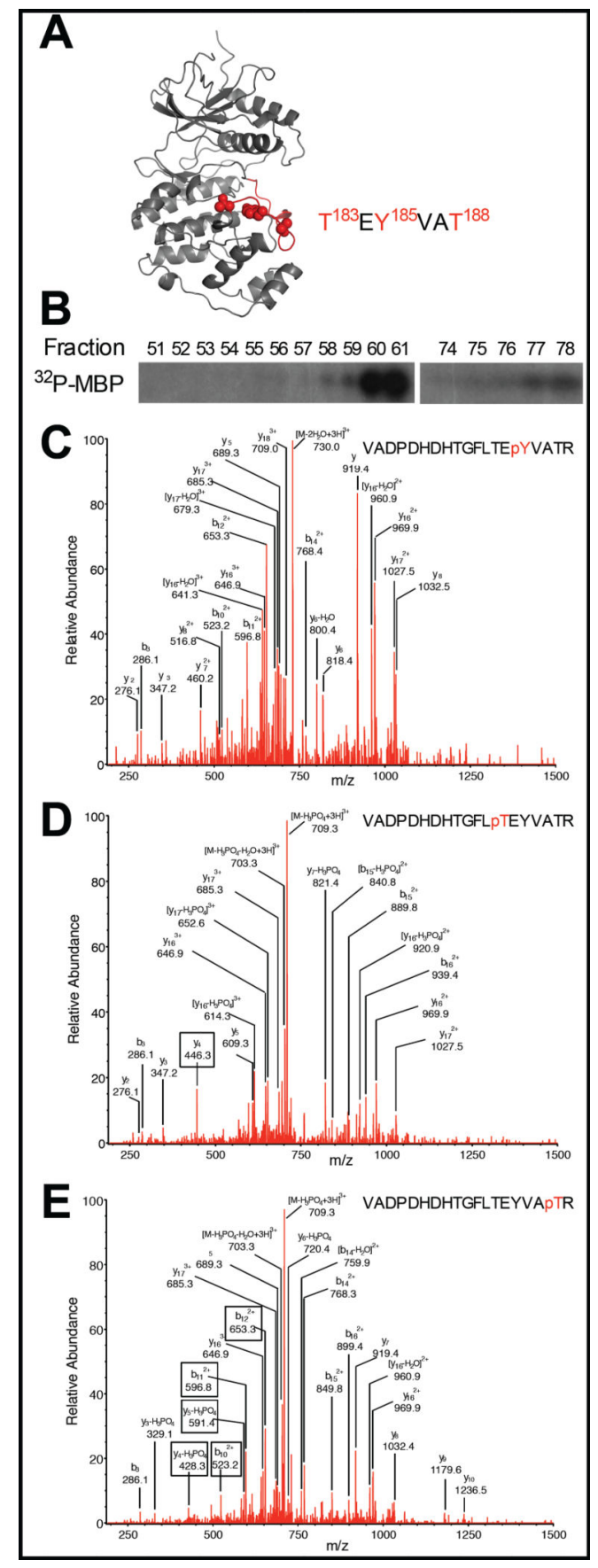

Figure 1.

Anion exchange chromatography of rat ERK2 and subsequent mass spectrometry (MS) analysis reveals multiple phosphorylation and activity states. (A) Low-activity structure of ERK2 with detected activation loop phosphorylation sites indicated in red [PDB: 1ERK]. (B) 6XHis-ERK2 from a nickel column was purified further on MonoQ. Individual fractions were assayed for kinase activity. (C-E) Multiple phosphoforms were identified by MS of pooled fractions. Representative of three experiments. Three activation loop residues were found to be phosphorylated: Y185 (D), T183 (E), and T188 (F). 


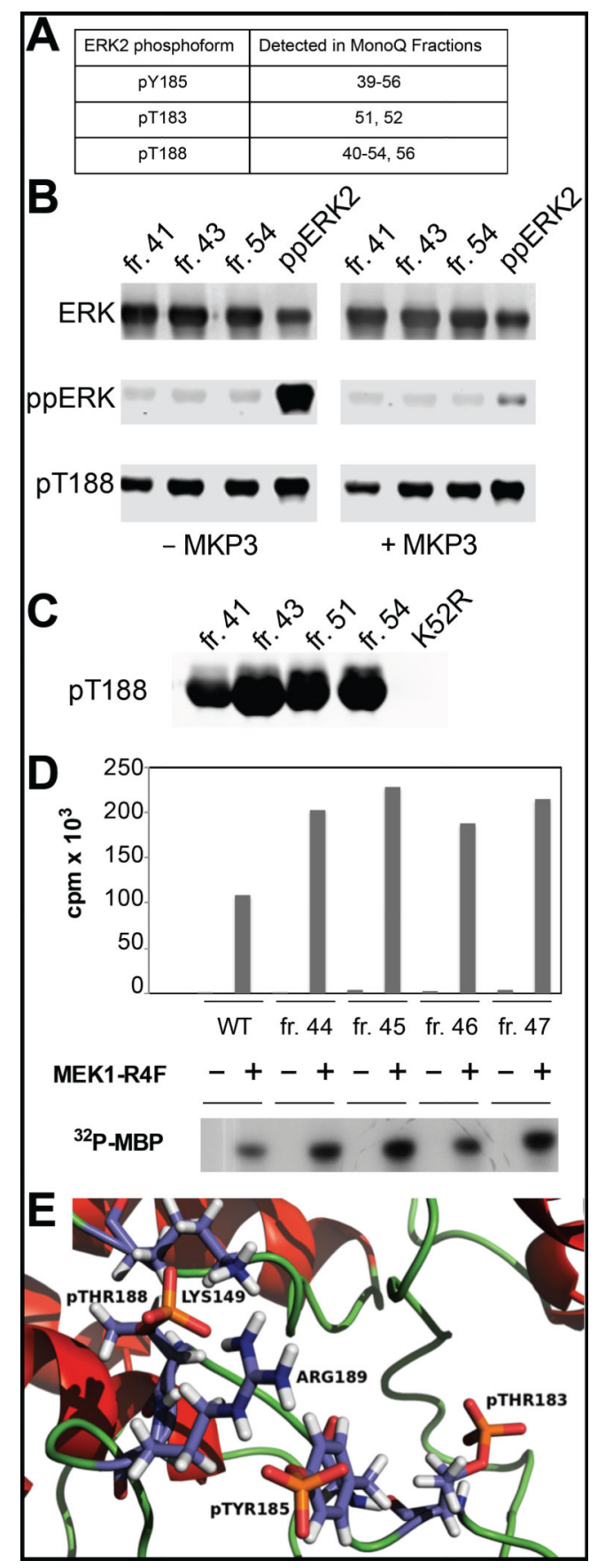

Figure 2.

Phosphorylated T188 (pT188) is present in bacterial preparations of ERK2. (A) Summary of MS analysis of fractions from a MonoQ purification of ERK2. (B) Selected fractions were immunoblotted with antibodies that recognize ERK2 regardless of its phosphorylation state, activated ERK2 (ppERK2), and pT188 selectively ${ }^{15}$. MAP kinase phosphatase 3 (MKP3) is able to dephosphorylate pY185 and pT183 but not pT188. (C) Immunoblot of MonoQ fractions of purified wild type ERK2 and a preparation of a kinase-dead ERK2 (K52R) with a pT188-selective antibody. (D) MEK1R4F recognizes and phosphorylates eluted fractions 
containing pT188, increasing ERK2 kinase activity toward MBP. (E) Model of the triply phosphorylated ERK2 activation loop. 


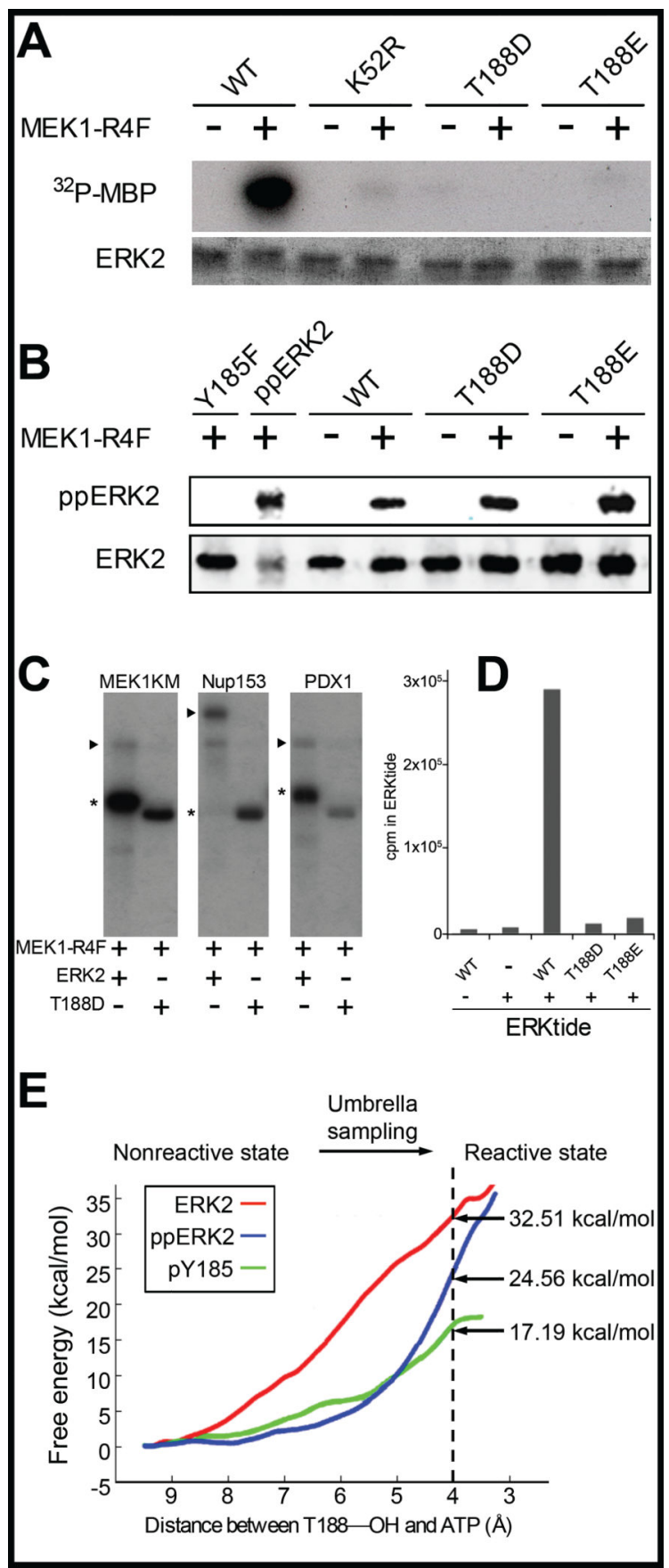

Figure 3.

Analysis of activities of ERK2 and T188 phosphomimetic substitutions. (A) MBP kinase assay with wild type ERK2 and kinase-dead ERK2 K52R compared to ERK2 T188D and T188E. (B) MEK1R4F phosphorylation of ERK2 and mutants. Recombinant Y185F is phosphorylated less well by MEK127. (C) Comparison of ERK2 or T188D kinase activities following activation by MEK1R4F with known ERK2 substrates. Phosphorylated substrates are denoted by wedges and phosphorylated ERK2 and T188 by asterisks. (D) Kinase activities of ERK2 T188D and T188E towards ERKtide. (E) Free energy profiles for ERK2 
T188 migration from equilibrium position to a more ATP-P $\gamma$-proximal position construed by molecular dynamics analysis. 


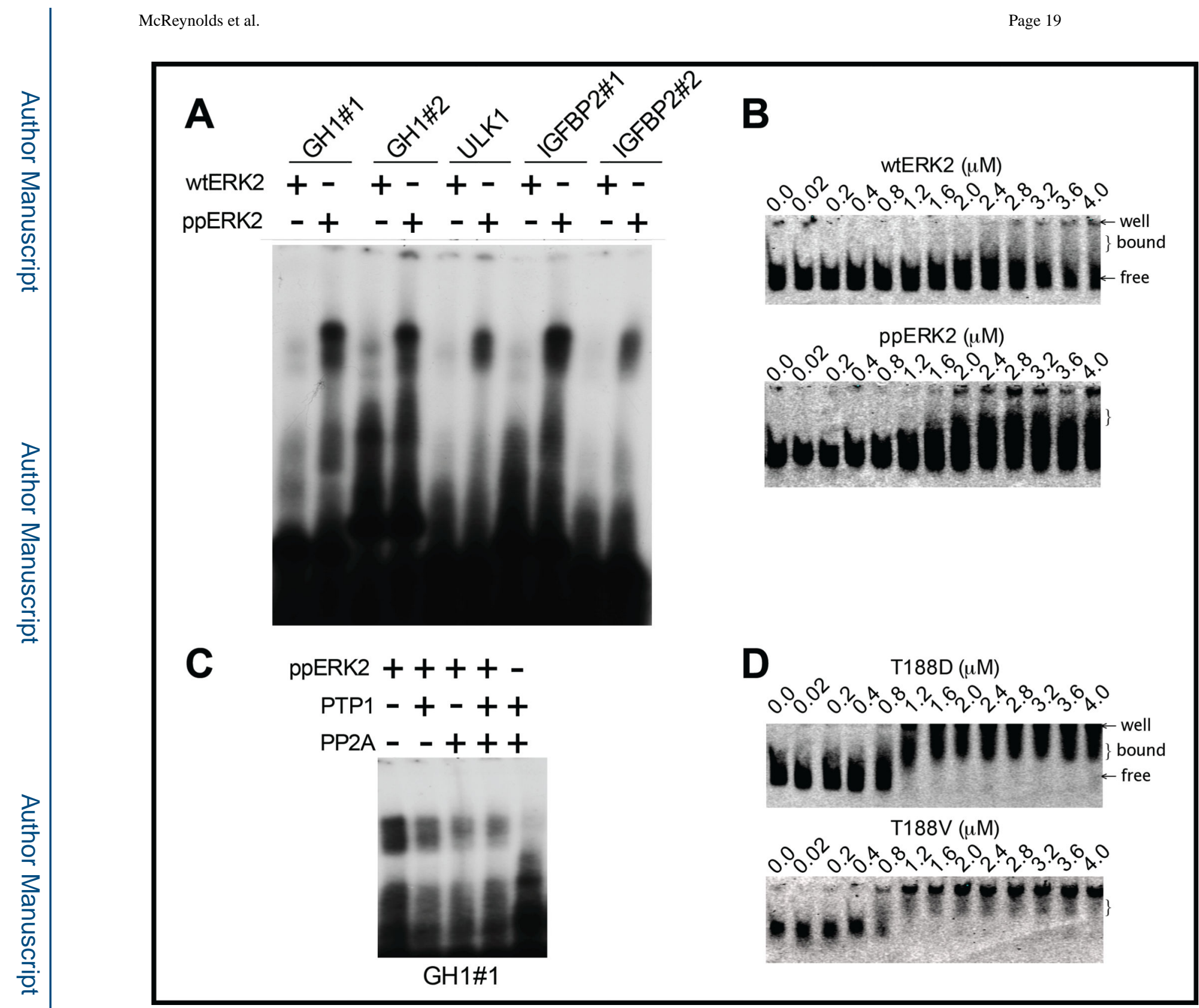

Figure 4.

ERK2 binds multiple oligonucleotides. (A) Gel shift assay with ERK2 and activated doubly phosphorylated ERK2 with radiolabeled sequences from GH1, ULK1, and IGFBP2 genes.

(B) Gel shift assays with ERK2 and ppERK2 and a trace amount of fluorescently labeled oligonucleotide $(100 \mathrm{pM})$ that maps to the insulin promoter and contains the motif GAAAC.

(C) Dephosphorylation with protein phosphatases PTP1 (phosphotyrosine-selective) and PP2A (phosphoserine/threonine-selective) reduces the interaction between ppERK2 and the GH1 oligonucleotide. (D) Gel shift assays with a trace amount of fluorescently labeled oligonucleotide (mIns19) (100 pM) with T188D and T188V. 